those of controls, $\mathrm{p}<0.05$. The expression of IL-23 protein were also higher than those of controls, which in concordance with the changes of mRNA.

Conclusions Our data show that local significantly increased levels of IL- 23p19mRNA in myocardium and IL-23 may play a role in the pathogenesis of mice virus myocarditis.

\section{e0217 STUDY ON THE PROTECTIVE EFFECT OF THE MIXTURE OF SHENGMAI PULVIS AND DANSHEN DECOCTION ON THE MYOCARDIUM OF TYPE 2 DIABETIC CARDIOMYOPATHY IN RATS MODELS}

doi:10.1136/hrt.2010.208967.217

${ }^{1}$ Niqing, ${ }^{2}$ Wangjie, ${ }^{2} Z$ hao An-bin, ${ }^{2}{ }^{2}$ B Bin, ${ }^{2}$ Wang Min, ${ }^{2}$ Huang Chun-rong, ${ }^{2}$ Li En-qing. ${ }^{1}$ Guang'anmen Hospital of China Academy of Chinese Medical Science, Beijing, China; ${ }^{2}$ School of Medicine, Jinan University, China

Objective To study the effect of the Mixture of Shengmai Pulvis and Danshen Decoction in protecting rats of the type 2 diabetic cardiomyopathy (DCM) model.

Methods 42 SD rat models of DCM, established by combination of insulin resistance by a high-fat diet with intraperitoneal injection of high dose streptozotocin $(50 \mathrm{mg} / \mathrm{kg}$ ), were evaluated in the damage of the myocardium by ECG at the twelveth week after modelling, and the serum are analysed for blood glucose (GLU), cholesterol and triglyceride (TG); the content of the left cardiac ventricle myocardial collagen was quantified by Masson staining test; the level of myocardial cell apoptosis was tested with Tunel apoptosis kit; the damage extent of the myocardial subcellular structure was observed by electron microscopy; the expression levels of cardiac TSP-1, TGF- $\beta 1$ and TRB-3 proteins were detected by immunohistochemistry, the changes of the expression levels of the cardiac TSP-1, A-TGF $\beta 1$ and L-TGF- $\beta 1$ protein were detected by Western blotting; and the changes of the mRNA expression levels of TSP-1 and TRB-3 were detected by real-time quantitative PCR.

Results Compared with the control group, the rat blood glucose, cholesterol, triglycerides were significantly decreased; the myocardial tissue was less damaged and the collagen fibre content was reduced in the group of the Mixture of Shengmai Pulvis and Danshen Decoction; The myocardial sub-cellular structural damage in electron microscopy was to a lesser extent, the expression levels of the myocardial TSP-1, TGF- $\beta 1$ and TRB-3 by immunohistochemical detection and the average expression levels of the myocardial TSP-1, A-TGF $\beta 1$ and L-TGF- $\beta 1$ by Western blotting were decreased; and the expression levels of TSP-1mRNA and TRB-3 mRNA by PCR detection were decreased than those of the control group.

Conclusion The Mixture of Shengmai Pulvis and Danshen Decoction can inhibit through multiple pathways the process of myocardial fibrosis in the rat myocardium of diabetic cardiomyopathy, and significantly delay the formation course of diabetic cardiomyopathy in hyperglycemia rats.

\section{e0218 BIOLOGICAL CHARACTERISTICS RESEARCH OF STENT COATING WITH ZEDORAY CONSTITUENTS IN A PORCINE MODEL}

doi:10.1136/hrt.2010.208967.218

Fu-Hai Zhao, Da-Zhuo Shi, Jian-Gang Liu, Pei-Li Wang, Da-Wu Zhang, Lei Zhang, JianPeng Du. Cardiovascular Center of Xiyuan Hospital Affiliated to China Academy of Chinese Medical Sciences, Beijing, China

Background In-stent restenosis is caused by neointimal hyperplasia, which involves abnormal growth of vascular smooth muscle cells
(VSMC). Zedoray constituents is known to inhibit smooth muscle cell hyperplasia and migration, while inhibit $\mathrm{ADP}$ induced platelet aggregation.

Objective To evaluate the biological characteristics of stents coating with zedoray constituents in porcine coronary model.

Methods Bare metal stents (BMS, $\mathrm{n}=36$ ), Sirolimus eluting stents (SES, $\mathrm{n}=36$ ) and Zedoray eluting stents (ZES, $\mathrm{n}=36$ ) were implanted in the proximal segment of three different epicardial coronary arteries in 36 swines randomly. Coronary angiography, optical coherence tomography (OCT) and histomorphologic analysis were performed at 30 days and 90 days after the procedure.

Results The 30 day $(n=24)$ OCT examination showed ZES arm has larger lumen diameter (LD), acceptable mean lumen stenosis of area (MSA) compared with BMS (LD: ZES $1.9 \pm 0.51 \mathrm{~mm}$, SES $1.85 \pm 0.41 \mathrm{~mm}$, BMS $1.1 \pm 0.3 \mathrm{~mm}, \mathrm{p}<0.05 ; \mathrm{MSA} \%$ : ZES $21.7 \pm 19.3$, SES 23.2 \pm 18.9 BMS 41.7 $\pm 21.3, p<0.001)$. By histomorphomeric analysis, similar injury scores were observed at the three arms $(p>0.05)$. However, significant inflammation score reduction was seen in ZES group (ZES: 0.65 \pm 0.54 , SES: $1.03 \pm 0.44$, BMS: 0.94 \pm 0.75 , $\mathrm{p}<0.001)$ compared to other two groups at 30 day, no differences in three groups at 90 day. Either at 30 day or 90 day, by qualitative analysis, well developed endothelium was seen in ZES arm, while impaired endothelium was observed with part of stent strut naked at vessel lumen at SES arm.

Conclusion Zedoray eluting stents can reduce neointimal hyperplasia with good endothelia coverage in porcine coronary model.

\section{e0219 STUDY ON THE ROLE OF CD4+CD25+TREG ON ATHEROSCLEROSIS IN APOE-/- MICE}

doi:10.1136/hrt.2010.208967.219

Xuemei Li, Yujie Li, Dongdan Zheng, Hao Tang, Mingxiang Wen. The First Affiliated Hospital, SUN Yat-sen University, Guangzhou, China

Objective To investigate the mechanism of $\mathrm{CD} 4{ }^{+} \mathrm{CD} 25^{+}$treg in atherosclerosis in ApoE-/- mice.

Methods 28 weeks-old male $\mathrm{ApoE}^{-/-}$mice were randomly divided into two groups feeding high-fat diet (AH) or normal diet (AN). Ten C57BL/6J male mice feeding normal-diet (BN). After 12 weeks, the whole aorta from the root to crotch of iliac artery was separated and the whole blood were centrifugated to get the serum. Paraffin sections of aorta were stained with $\mathrm{H} \& \mathrm{E}$ and morphometric parameters were measured using Image Pro Plus 6.0 system. Immunohistochemical stain was performed to verify the expression of the Foxp3 ${ }^{+}$and $\mathrm{CD}_{2} 5^{+}$cells in atherosclerotic tissue (\%). The ratio of $\mathrm{CD} 4^{+} \mathrm{CD} 25^{+} / \mathrm{CD} 4^{+}$in spleen was calculated by Flow Cytometry. The serum concentration of TGF- $\beta 1$, IL-10 were detected by ELISA. SPSS13.0 was used to analyse the data. All values were expressed as Mean \pm SD. Independent-Samples $T$ Test was applied to compare two samples of quantitative data after testing their normality. $\mathrm{p}<0.05$ was considered statistically significant.

Results There were advanced atherosclerotic plaques in $\mathrm{ApoE}^{-/-}$ mice, but in C57BL/6J. The thickness of intima $(\mu \mathrm{m})$, plaque area $\left(\mu \mathrm{m}^{2}\right)$, plaque/lumen ratio in AN group were significantly higher than in $\mathrm{BN}$ group $(12.24 \pm 1.34$ vs $7.33 \pm 2.23,600265.4 \pm 263876.25$ vs $0,15.93 \pm 5.45$ vs 0 , all $p<0.01)$. So did in $A H$ group than that in $A N$ group (all $\mathrm{p}<0.05)$. The percentage of spleenic $\mathrm{CD} 4^{+} \mathrm{CD} 25^{+}$treg $(\%)$ and the serum concentration of TGF- $\beta 1$, IL- $10(\mathrm{pg} / \mathrm{ml})$ in AN group were significantly decreased than in BN group (9.4 \pm 4.00 vs $13.8 \pm 3.97$, $116.05 \pm 32.27$ vs $191.27 \pm 95.27,41.83 \pm 16.15$ vs $61.84 \pm 23.05$, all $\mathrm{p}<0.05)$. These in $\mathrm{AH}$ group significantly reduced than in AN group $(5.8 \pm 1.51$ vs $9.4 \pm 4.00,83.97 \pm 33.45$ vs $116.05 \pm 32.27,27.50 \pm 11.54$ vs $41.83 \pm 16.15$, all $\mathrm{p}<0.05$ ). There were no Foxp3 ${ }^{+}$and $\mathrm{CD}^{2} 5^{+}$cells in plaques-free intima in $\mathrm{BN}$ group. The expression of Foxp3, CD25 $5^{+}$cells were significantly decreased in AH group than in AN group $(1.28 \pm 1.20$ vs $3.04 \pm 1.92,2.00 \pm 1.39$ vs $3.98 \pm 1.67$, all $p<0.05$ ). 
Conclusions The spleenic $\mathrm{CD} 4{ }^{+} \mathrm{CD} 25^{+}$treg and serum concentration of TGF- $\beta 1$, IL-10 of mice were decreased and there were lower frequency of Foxp $3^{+}$and $\mathrm{CD} 25^{+}$cells in severe atherosclerotic plaques than in mild. It meant that $\mathrm{CD} 4{ }^{+} \mathrm{CD} 25^{+}$treg may has antiatherosclerotic role on atherosclerotic progression.

\section{e0220 THE INVOLVEMENT OF IL-23/TH17 PATHWAY IN MURIN MODEL OF COXSACKIE VIRUS B3-INDUCED VIRAL MYOCARDITIS}

doi:10.1136/hrt.2010.208967.220

Fan Yang, Weifeng Wu, Yuluan Yan, Qing Kong, Yu Pang. Department of Cardiology, The First Affiliated Hospital of Guangxi Medical University, Nanning, China

Background The IL-23/Th17 pathway plays an important role in the development of chronic inflammatory diseases and autoimmune diseases. However, the role of the IL-23/Th17 axis in the regulation of virus myocarditis (VMC) is still largely unknown.

Methods VMC was induced in male $\mathrm{Balb} / \mathrm{c}$ mice by CVB3 peritoneal injection. Mice injected with PBS were taken as the controls. IL-23, IL-17 and ROR $\gamma \mathrm{t}$ mRNA in the myocardium of VMC were assessed by semi-quantitative RT-PCR on the time of $0,1,2,3,4$ and 6 weeks after injection. IL-23, IL-17 protein from blood plasma was evaluated by ELISA. Flow cytometric analysis was used to evaluate the frequencies of Th17 subsets in $\mathrm{CD}^{+}{ }^{+}$Tcell. CD4+ $\mathrm{T}$ cells were isolated from VMC mice and cultured with rIL-23 in vitro to investigate the function of IL-23 in the IL-23/Th-17 pathway.

Results Comparing with the controls, IL-23, IL-17 and ROR $\gamma$ t mRNA were steadily expressed in the myocardium of infected mice from 1 week after virus injection $(\mathrm{p}<0.01)$, IL-23 and IL17 protein level increased from 1 st week to 6 th week. The frequencies of Th17 cells were obviously increased in VMC mice 1 week after infection $(p<0.01)$, the maximum level of Th17 cells was reached at 4th week. The ratio of Th17 cells in the spleen lymphocyte significantly improved after rIL-23 stimulation, the IL-17 and ROR $\gamma \mathrm{t}$ mRNA expression of the cultured cells and the IL-17 protein in the culture supernatants increased after rIL-23 stimulation $(\mathrm{p}<0.05)$.

Conclusions IL-23/Th-17 pathway may play an essential role in VMC

\section{e0221 EFFECTS OF EXTRACORPOREAL CARDIAC SHOCK WAVE THERAPY ON EXPRESSION OF ENDOTHELIAL NITRIC OXIDE SYNTHASE AND BASIC FIBROBLAST GROWTH FACTOR IN SWINE WITH ACUTE MYOCARDIAL INFARCTION}

doi:10.1136/hrt.2010.208967.221

${ }^{1}$ Shuai Sun, ${ }^{2}$ Tao Guo. ${ }^{1}$ Department of Cardiology, First People's Hospital of Yunnan Province, Kunming, Yunnan, China; ${ }^{2}$ Department of Cardiology, First Affiliated Hospital of Kunming Medical College, Kunming, Yunnan, China

Objective To observe the effects of extracorporeal cardiac shock wave therapy (CSWT) on cintent of endothelial nitric oxide synthase (eNOS) and basic fibroblast growth factor (bFGF) in serum and myocardial tissue in swine with acute myocardial infarction (AMI).

Methods 12 Model swines with acute myocardial infarction was made, and were randomly divided into two groups with six in each group: simplex myocardial infarction as the control group and the experimental group which received CSWT treatment. (three times on the first, third, fifth days after operation. 200 hit/point. Total: 12 points. Energy: $0.09 \mathrm{~mJ} / \mathrm{mm}^{2}$ ). Peripheral blood was extracted at eight different time points before and after operation (immediate, the first, third, fifth days, 1, 2, 3, 4 weeks after operation) to detect serum eNOS content with enzymelinked immunosorbent assay.
Materials at myocardial tissue from the swines killed 1 month later were obtained to detect expression amount of eNOS and bFGF with semi quantitative RT-PCR method.

Results eNOS rose up in the experimental group 1 day after CSWT and a reached the peak on the fifth day, whereas eNOS gradully reduced in the control group, which slowed significant difference between the two groups $(p<0.01)$. Detection with semi quantities RT-PCR of myocardial tissues of infarction border area showed that eNOS and bFGF expression in the experimental group were obviously higher than those in the control group ((eNOS 27.705 \pm 4.13 ) vs $(16.448 \pm 3.21)$ (bFGF $32.571 \pm 4.23)$ vs $(17.858 \pm 4.17 \mathrm{p}<0.01))$.

Conclusions Extracorporeal cardiac shock wave therapy of acute myocardial infarction can effectively promote rise of eNOS and bFGF, which may be a new way to cure AMI.

\section{e0222 EFFECTS AND FUNCTION MECHANISM OF HYDROGEN SULFIDE ON MYOCARDIAL ISCHAEMIA REPERFUSION ARRHYTHMIA IN RATS}

doi:10.1136/hrt.2010.208967.222

${ }^{1}$ Chen Xiehui, 'Li Peng, ${ }^{1}$ Huang Xiaoping, ${ }^{2}$ Wang Dongming, ${ }^{2}$ Chen Songming, ${ }^{1}$ Yang Zeming, ${ }^{1}$ Chen Zhifan, ${ }^{1} X u$ Wenmin, ${ }^{1}$ Chen Liping. ${ }^{1}$ The 2nd People's Hospital of Futian District, Shenzhen, Guangdong, China; ${ }^{2}$ The First Affiliated Hospital of Shantou University Medical College, Shantou, Guangdong, China

Objective To explore the Effects and Function Mechanism of hydrogen sulfide on Myocardial Ischaemia reperfusion Arrhythmia in Rats.

Methods We used sodium hydrosulfide (NaHS) as the donor of $\mathrm{H}_{2} \mathrm{~S}$, $\mathrm{SD}$ rats were randomly divided into sham group, Myocardial Ischaemia reperfusion group (IR group), IR $+\mathrm{NaHS}$ group, and IR + $\mathrm{NaHS}+$ glibenclamide group. We monitor the Haemodynamics of rats, including heart rate, arterial pressure, left ventricular pressure et al. We also observe the rate of ventrical arrhythmia in each group. Result $\mathrm{H}_{2} \mathrm{~S}$ can significantly reduces rats' heart rate, arterial pressure and left ventricular pressure. It also reduces the rate of ventrical arrhythmia in Myocardial Ischaemia reperfusion Rats. The $\mathrm{K}_{\text {ATP }}$ Channel Blocker glibenclamide can weaken the $\mathrm{H}_{2} \mathrm{~S}$ ' Antiarrhythmic effects $(\mathrm{p}<0.01)$.

Conclusions $\mathrm{H}_{2} \mathrm{~S}$ can reduces the rate of ventrical arrhythmia in Myocardial Ischaemia reperfusion Rats. The Function Mechanism may be associated with the $\mathrm{K}_{\text {ATP }}$ signal transduction pathway in cells.

\section{e0223 EFFECTS OF NEOTYPE PERITONEAL COOLING ON THE INJURED OF INTESTINAL MUCOUS AFTER CARDIOPULMONARY RESUSCITATION IN RABBITS}

doi:10.1136/hrt.2010.208967.223

Xuan Dai, Xiao-xing Liao, Chun-Lin Hu, Hong-Yan Wei, Xin Li, Hong Zhan. Department of Emergency Medicine, The First Affiliated Hospital of Sun Yat-Sen University, Guangzhou, China

Objective To explore whether the peritoneal cooling after cardiopulmonary resuscitation could improve the injured of intestinal mucous in rabbits.

Methods 36 adult New Zealand rabbits were induced ventricular fibrillation by $\mathrm{AC}$ current. After the restore of spontaneous circulation (ROSC), rabbits were randomly divided into three groups according to the way of body temperature controlling, that is nomothermia group (NT), surface cooling group (SC) and peritoneal cooling group (PC). The changing of tympanic temperature and peritoneal temperature were observed after ROSC. The animals were sacrificed by over anaesthesia after ROSC for $12 \mathrm{~h}$, the end ileum was removed and fixed in formalin, the histological injured 